\title{
Man versus Machine: Comparing manual with LIWC coding of perceptual and contextual details for verbal lie detection
}

Short title: Comparing manual with LIWC coding

working paper, version May, 2, 2021

\section{$\underline{\text { Authors and affiliations }}$}

Manon Schutte ${ }^{1}$, Glynis Bogaard ${ }^{2}$, Erik Mac Giolla ${ }^{3}$, Lara Warmelink ${ }^{4}$, Bennett Kleinberg ${ }^{5}$, and Bruno Verschuere*1

\footnotetext{
${ }^{1}$ University of Amsterdam

${ }^{2}$ Maastricht University

${ }^{3}$ University of Gothenburg

${ }^{4}$ Lancaster University

${ }^{5}$ University of Tilburg

* Bruno Verschuere, Postbus 15933, 1001 NK Amsterdam, The Netherlands, (email:

b.j.verschuere@uva.nl)
} 


\section{Abstract \\ (max 250 words; current word count: 226 words)}

Purpose: Truthful statements are theorized to be richer in perceptual and contextual detail than deceptive statements. The level of detail can be coded by humans or computers, with human coding argued to be superior. Direct comparisons of human and automated coding, however, are rare.

Methods: We applied automatic identification of details with the Linguistic Inquiry and Word Count (LIWC) software on truthful and deceptive statements from four datasets that had been manually coded for details.

Results: We noted that the common way of scoring manual and LIWC coding hampers a direct comparison because they rely on different metrics - count and proportion scores, respectively. Lie-truth differences varied substantially across metric and dataset (LIWC: $0.09 \leq$ Cohen's $d \leq .89$; Manual: $0.03 \leq$ Cohen's $d \leq .80$ ). When set to the same metric, neither method seemed to outperform the other. Using count scores, both LIWC and manual coding indicated that truthful statements about past events contain more perceptual and contextual details than deceptive statements. Across the four datasets, we also observed considerable variation in manual coding.

Conclusions: Human coding does not necessarily outperform LIWC coding of perceptual and contextual details in discriminating lies from truths. Our findings call for systematic comparison of human and automated verbal lie detection approaches on the same data, and we reiterate the need for better data sharing practices to help accomplish that aim.

\section{$\underline{\text { Keywords }}$}

Coding, CBCA, Reality monitoring, Verifiability approach, Details 
$\underline{\text { Data availability statement }}$

The data that support the findings of this study are openly available at https://osf.io/75hsp/. 
Man versus Machine:

Comparing manual and LIWC coding of details for verbal lie detection

To try and discern lies from truths, contemporary verbal lie detection research often relies on the amount of perceptual and contextual details in a statement (Nahari, 2018; Vrij, 2019). Perceptual details concern what was seen, heard, felt, smelled or tasted. Contextual details, on the other hand, concern the temporal and spatial orientation of events, such as times, dates, locations, but also the order of activities, or a description of who was where in a room. Reality Monitoring theory (RM; Johnson \& Raye, 1981; Masip, Sporer, Garrido, \& Herrero, 2005) predicts that truthful statements will contain more perceptual and contextual details than deceptive statements. The rationale behind that prediction is that truth tellers have actually experienced the event and, therefore, can rely on a memory trace that is richer in perceptual and contextual detail. Identifying perceptual and contextual details has been done either by humans or by computers, but direct comparisons of manual and automated coding, however, are rare. Our study begins to fill this void by comparing the manual coding of perceptual and contextual details with automated coding of these details using the Linguistic Inquiry and Word Count (LIWC) software (Pennebaker, Boyd, Jordan, \& Blackburn, 2015) on four datasets.

Perceptual and contextual details in a statement are typically identified by trained human coders. Using a dedicated coding scheme, the coders go through the statements and manually mark every piece of information that qualifies as perceptual or contextual detail. For instance, a human may code the sentence 'At $2 \mathrm{pm}$, the screaming started' to contain one perceptual detail (i.e., 'the screaming', which is something the narrator has heard) and one contextual detail (i.e., 'At 2pm', which specifies the time of the event). A possible advantage of that manual approach is that they can take into account the context of the statement, as well as legal psychological theory and findings. For instance, humans often dismiss repetitions. A 
human coder will dismiss not only a second mentioning of 'Bruno's Beer Bar' but also referrals that use different wording but refer to the same person or object, so-called co-references (e.g., 'then we went back to the pub'). This flexibility in coding, however, reduces the standardization across studies. Moreover, there is no consensus about a standard scoring procedure (Nahari et al., 2019). Instead, a diverse pool of coding schemes is in use. The empirical evidence for the validity of manual coded perceptual and contextual details to discern lies from truths is mixed. This has been attributed to a lack of a standardized coding scheme (Masip et al., 2005; Vrij, 2008).

In principle, computer scoring could be a cost efficient and standardized alternative to manual coding. One popular tool to automatically code statements is the Linguistic Inquiry and Word Count (LIWC) software (Pennebaker, Boyd, Jordan, \& Blackburn, 2015). LIWC uses a dictionary approach, recognizing (certain) words, and evaluating whether they belong to a predetermined category. For instance, the words love, nice, and sweet belong to the category positive emotion, and by categorizing these words, LIWC aims to provide an indication of how much positive affect the statement expresses. This example also illustrates a key limitation of LIWC. In everyday language, nice does not necessarily reflect a positive emotion, but may also be neutral or even reflect negative emotionality (e.g., when used with irony).Its higher standardization therefore comes at the cost of rigidity, which may lower its validity in decoding mental states from text.

Bond and Lee (2005) reasoned that the LIWC categories senses (including words such as call and speak), space (including words such as under and north), and time (including words such as until and noon), may provide an automated method of scoring perceptual details (category 'senses') and contextual details (categories 'space' and 'time'). They found mixed support for their idea. True statements contained more LIWC-coded spatial details, but not more temporal details, and, in fact, fewer sensory and perceptual details. Also, a meta-analysis 
of 44 studies, half using LIWC, found that the average lie-truth effect for automated coding of categories related to perception, time, and space was close to zero (Hauch, Blandón-Gitlin, Masip, \& Sporer, 2015). This meta-analysis also showed effects were heterogeneous and pointed to potential moderators. For example, the prediction resulting from Reality Monitoring that truthful statements would contain more LIWC coded perceptual details than deceptive statements, was confirmed only when motivation was high, or the account was handwritten.

From these studies it is clear that both manual and automated coding have its advantages and disadvantages. Unfortunately, their accuracy in discerning lie from truth is now based upon different sets of studies, leading to an apples vs oranges comparison. There is a paucity of studies providing a direct comparison of manual versus automated coding in lie-truth discrimination on the same statements. In a rare exception, Vrij, Mann, Kristen, and Fisher (2007) instructed 120 students to lie or tell the truth about a mock theft during an interview conducted in either the information-gathering, accusatorial or behavioral analysis style interview. Statements were coded on five RM criteria (visual details, auditory details, temporal details, spatial details, and cognitive operations) that were combined into an RM total score, and were coded on four LIWC categories (senses, time, space, and cognitive mechanisms). The RM score showed large lie-truth effects in the information-gathering $\left(d=0.71^{1}\right)$ and behavioral analysis style interview $(d=1.17)$, but, as anticipated by the authors, not in the accusatory interview $(d=0.08)$. Lie-truth discrimination differed by interview type. For instance, effects were in line with the prediction for LIWC category "senses" in the behavioral analysis style interview $(d=0.80)$ and accusatory interview $(d=0.46)$, but showed an effect in the unpredicted direction in the information-gathering interview $(d=-0.74)$. Based on a single study, however, it seems premature to draw strong conclusions. In the present study, we

\footnotetext{
${ }^{1}$ Own calculations using the $M s S D$ s, and $n$ s reported in Table 1 of Vrij et al. (2007) and using $d_{\text {between }}$
} 
therefore gathered four datasets of manual coded statements to directly compare manual coding with LIWC coding.

\section{Method}

We asked several verbal lie detection researchers to provide us with datasets that contained truthful and deceptive statements manually coded for perceptual and contextual details. We obtained four datasets from four different laboratories (Bogaard, Colwell, \& Crans, 2019; Warmelink, Vrij, Mann, Jundi, \& Granhag, 2012; Mac Giolla, Ask, Granhag, \& Karlsson, 2019; Verschuere, Schutte, van Opzeeland, \& Kool, 2021). A detailed description can be found in the references provided, but below we list the key features of the datasets. Coding scheme and data for the present study can be found on https://osf.io/75hsp.

\section{$\underline{\text { Bogaard et al. (2019) dataset }}$}

For full details, we refer to Bogaard et al. (2019). In brief, 100 students from Maastricht University were asked to provide both a truthful account about an emotional event that they had really experienced and that lasted a brief and discrete period of time, and, based on a realistic scenario, a deceptive account about an event that had never happened. The order of truthful and deceptive accounts was counterbalanced.

Students were interviewed either via a Reality Interview, based on mnemonic strategies, or a Free Recall interview, which solely consisted of freely recalling the event. The first question of the two interview conditions was the same (i.e., 'Can you describe the event in as much detail as possible?') and is the focus of the current analyses. In supplementary analyses, we split the data per interview type.

Statements were on average 159 words long ( $S D=112.89$; range: 22 - 743 words). Each statement was coded on 19 CBCA and eight RM criteria by two raters, who were familiar 
with the background literature and had received $3 \mathrm{~h}$ of training and practice in coding. Our analysis focuses on three RM criteria: Perceptual details (defined as the amount of sensory information; e.g., 'I look around'), spatial details (defined as the amount of information about places and locations; e.g., 'at my house'), and temporal details (defined as the amount of timerelated information; e.g., 'Initially'). A proportion of the statements $(25 \%)$ was coded by the second rater. The intraclass correlation coefficient was 0.83 for perceptual details, 0.89 for spatial details, and 0.98 for temporal details. As an index of perceptual and contextual details, we summed perceptual, spatial and temporal details.

\section{Warmelink et al. (2012) dataset}

For full details, we refer to Warmelink et al. (2012). In brief, 86 Portsmouth University students and employees were asked to provide either a truthful statement about a trip they had planned in the near future or a deceptive statement where they pretended they planned to travel to a certain destination for a certain purpose. The destination and purpose were provided by the experimenter and matched to a truthful account.

The interview consisted of 26 questions divided into four parts: Five general questions (e.g., "What is the main purpose of your trip"), seven core event questions (e.g., "What is the most important place you'll go to on your trip?"), seven questions about Transportation (e.g., "How long will the journey take?"), and seven questions about the planning of the trip (e.g., "Please tell me in which order you did the planning"). The interview was identical for all participants, except for two extra questions in the General category addressed to participants who had expressed they had previously been at the destination. Thus, in the present study we used the responses of the interviewees on the 26 questions (the questions can be found on https://osf.io/hyjse/). 
Statements were on average 1062 words long $(S D=850.68$; range: 288 - 6284 words).

All statements were coded by one coder on several categories. We combined spatial details (defined as any information on what's where; e.g., 'there', 'up the top'), temporal details (defined as any information on time or order; e.g., 'then', 'after two hours') and visual, auditory, olfactory, taste, and tactile details into an index of perceptual and contextual details. A proportion $(10 \%)$ of the statements were also coded by a second coder. The correlations between the two coders was $r=0.88$ for spatial details, $r=0.98$ for visual details (reliability for the other categories that we selected was not provided).

\section{Mac Giolla et al. (2019) dataset}

For full details, we refer to Mac Giolla et al. (2019). In brief, 100 participants (primarily university students) partook in the Gothenburg study. Fifty of them had planned a trip abroad in the near future. In a pre-questionnaire they were asked about their destination, purpose, and travel details. The other fifty participants (the liars) were each provided with the brief trip description from their matched truth teller. This served as a framework to base their deceptive interview on. All participants were invited to the la and interviewed face to face. The interview was set up to resemble a visa interview, which started by asking the participants to describe their future trip in as much detail as possible. In the present study, we analyzed the response to that first question.

Statements were on average 165 words long ( $S D=131.66$; range: 15 - 859 words). After a training session, one coder rated the statements on the twelve RM categories. A second coder rated $15 \%$ of the statements. As an index of perceptual and contextual details, we combined visual, auditory, olfactory, taste, tactile, temporal and spatial details. The inter-rater reliability was $r=1.0$ for spatial information, $r=.41$ for temporal information, and not reported for the perceptual categories. 


\section{$\underline{\text { Verschuere et al. (2021) dataset }}$}

For full details, we refer to Verschuere et al. (2021). In brief, 72 students from the University of Amsterdam were asked to provide an account of what they did in the past 15 minutes. Truth tellers $(n=38)$ had just engaged in their regular campus activities on several locations of the campus. Liars $(n=34)$ had just engaged in a mock crime, and falsely stated they too had only engaged in their regular campus activities.

Students were informed that an investigator would check whether the details provided in their statement could be verified and were encouraged to provide a convincing statement. The statement was handwritten. The corresponding instruction was to describe their activities in the past 15 minutes in as much detail as possible.

Statements were on average 168.60 words long ( $S D=89.03$; range: 55 - 568 words). Each statement was coded by two raters, who were familiar with the background literature and had received $2 \mathrm{~h}$ of training and practice in coding. The raters coded for spatial (i.e., information about spatial arrangements of things, people and locations), perceptual (i.e., details about what the participant saw, felt, heard or smelt), and temporal (i.e., about the order of events or the timing of an event) details. Subsequently, these details were coded for verifiability, but we ignore the distinction between verifiable and unverifiable details in the present study. The two raters discussed their coding with a third investigator who helped reach a consensus in case of disagreements. We use the consensus scoring.

All statements $(100 \%)$ were coded by the two raters. The intraclass correlation coefficient was 0.87 for perceptual and contextual details. As an index of perceptual and contextual details, we summed perceptual, spatial and temporal details.

\section{$\underline{\text { LIWC coding }}$}


The original transcripts of the four datasets were first cleaned from experimenter questions, filler words such as "ehm", "uh", and "haha", and from referrals to participants' behaviour such as [laughs] or [sighs].

The cleaned transcripts were analysed with the Linguistic Inquiry and Word Count Software. The built-in standard English dictionary (2015) was used to analyse the Warmelink et al. (2012) dataset. For the Bogaard et al 2019 and Verschuere et al 2021 datasets, the Dutch 2015 library was used (Van Wissen \& Boot, 2017), and for the MacGiollaet al 2019 dataset we received a Swedish dictionary from a fellow researcher (https://pauljtaylor.com/data_code/swedish-liwc-dictionaries/).

The LIWC comprises approximately 90 word categories, ranging from linguistic dimensions such as pronouns and articles to psychological constructs (affect, cognition) and informal language markers. With a dictionary approach, it categorizes each word in a statement to the related category. The LIWC then scores for each statement the proportion of words belonging to each category (thereby by default correcting for statement length). So if a 100word text contains two numbers (e.g., six, and thirty-four), the score for the LIWC category will be 2\%. Following Bond and Lee (2005; see also e.g., Kleinberg et al., 2017) we summed the LIWC categories perceptual processes, space and time as an index for perceptual and contextual details.

Results

Analyses scripts can be found on https://osf.io/75hsp/

Lie-truth differences in manual (count score) and LIWC (proportion scores) coded perceptual $\underline{\text { and contextual details }}$ 
We first calculate manual and LIWC coding, the way they are typically calculated (see also Vrij et al., 2007), that is manual count scores and LIWC proportion scores. For the manual count of perceptual and contextual details in truth-tellers and liars statements, the effect size varied from $0.14 \leq d \leq 0.80$, and was statistically significant in two of the four datasets, see Table 1.. Table 1 further shows that for LIWC scored perceptual and contextual details, the effect size varied from $0.03 \leq d \leq 0.89$, and was statistically significant effects in two of the four datasets ${ }^{2}$.

Table1. Perceptual and contextual details in truthful vs deceptive statements: Manual count scores versus LIWC proportion scores

\begin{tabular}{|c|c|c|c|c|c|c|}
\hline & \multicolumn{3}{|c|}{$\begin{array}{l}\text { Manual coding } \\
\text { (count scores) }\end{array}$} & \multicolumn{3}{|c|}{$\begin{array}{l}\text { LIWC coding } \\
\text { (proportion scores) }\end{array}$} \\
\hline & Truth & Lie & $d$ & Truth & Lie & $d$ \\
\hline & $M(S D)$ & $M(S D)$ & {$[95 \% \mathrm{CI}]$} & $M(S D)$ & $M(S D)$ & {$[95 \% \mathrm{CI}]$} \\
\hline Bogaard et & 9.16 & 7.81 & 0.29 & 16.60 & 16.49 & 0.03 \\
\hline al 2019 & $(4.98)$ & $(4.15)$ & {$[0.01,0.48]$} & $(4.23)$ & $(4.02)$ & {$[-0.26,0.31]$} \\
\hline Warmelink & 241.45 & 210.95 & 0.22 & 15.40 & 13.66 & 0.89 \\
\hline et al 2012 & (149.36) & $(121.60)$ & {$[-0.21,0.65]$} & (1.93) & $(2.00)$ & {$[0.44,1.33]$} \\
\hline Mac Giolla & 2.18 & 1.94 & 0.14 & 17.75 & 15.36 & 0.50 \\
\hline et al 2019 & $(2.03)$ & $(1.42)$ & {$[-0.26,0.54]$} & $(4.68)$ & $(4.79)$ & {$[0.10,0.91]$} \\
\hline
\end{tabular}

\footnotetext{
${ }^{2}$ Interpretation of the LIWC findings is qualified by the observation that the LIWC scoring differed greatly with language. LIWC recognized the majority of the Dutch text (Bogaard et al., 2019: 85.71, Verschuere et al., 2021; 81.55\%) and the English text (Warmelink et al., $2012 ; 94.01 \%$ ) datasets, yet only $55.43 \%$ of the Swedish text (Mac Giolla et al, 2019), showing that the dictionaries differ in comprehensiveness.
} 
Verschuere

et al 2021
24.82

(10.11)
17.56

(7.80)
0.80

$[0.31 ; 1.29]$
22.35

20.48

0.45

(3.71)

Note: We used $d_{\text {within }}$ (based on Gibbons, Hedeker, \& Davis, 1993) for Bogaard et al 2019 as it relied on a within-subjects design. We used $d_{\text {between }}$ for the other datasets as they relied on a between-subjects design. Effect sizes were calculated with the effsize package in R (v0.7.6; Torchiano, 2019). A positive effect size $d$ indicates that the variable was higher in truth-tellers than liars, in line with RM predictions.

\section{The impact of statement length when comparing manual to LIWC coding}

When comparing the manual with the LIWC coding, it is important to note that LIWC by default relies on proportion scores, implying that the coding for details is automatically correcting for statement length. Given statement length can vary widely within and between interviewees and that truthful statements are often longer than deceptive statements (see Table 2), correcting for statement length can have a great impact on lie-truth effects. Elntib, Wagstaff, and Wheatcroft (2015) found that manual-coded RM criteria no longer differentiated between lies and truths after correcting for statement length (see also Elntib \& Wagstaff, 2017). This leads to the possibility that manual coding outperforms LIWC not because of higher validity but because of the use of count scores instead of proportion scores. To allow for an unbiased comparison of manual versus automated coding, we examined how controlling for word count impacted the comparison.

Table 2. Statement length in truthful vs deceptive statements for the four datasets.

\begin{tabular}{ccc} 
Truth & Lie & $d[95 \% \mathrm{CI}]$ \\
$M(S D)$ & $M(S D)$ & \\
\hline
\end{tabular}




\begin{tabular}{lccc} 
Bogaard et al & 173.72 & 144.81 & 0.25 \\
2019 & $(124.36)$ & $(98.62)$ & {$[0.09,0.45]$} \\
Warmelink et al & 1093.43 & 1032.66 & 0.07 \\
2012 & $(970.59)$ & $(728.30)$ & {$[-0.36,0.50]$} \\
Mac Giolla et al & 142.29 & 188.54 & -0.36 \\
2019 & $(106.45)$ & $(149.97)$ & {$[-0.76,0.05]$} \\
Verschuere et al & 183.66 & 151.76 & 0.36 \\
2021 & $(95.63)$ & $(79.03)$ & {$[-0.11 ; 0.84]$} \\
\hline
\end{tabular}

First, we calculated proportion scores for manual coding, allowing for a direct comparison with the LIWC proportion scores. We calculated the proportion of the statement that was manually qualified as a perceptual or contextual detail as frequency per 100 words (following Elntib et al., 2015) using the formula [(Number of perceptual and contextual details) / (Number of words)] x 100]. Table 3 shows that lie-truth effect sizes for manual proportion scores varied from $0.03 \leq d \leq 0.70$ and was statistically significant in only one of the four datasets. When both manual and LIWC scores were expressed as proportion scores, neither method seemed to systematically outperform the other, see Table 1 and 3.

Table 3. Perceptual and contextual details in truthful vs deceptive statements: Manual proportion scores.

Across True Lie Lie-truth Differences

statements

Cohen's d $[95 \% \mathrm{CI}]$ 
statemen statement

ts $\quad \mathrm{S}$

\begin{tabular}{lcccc}
\hline Bogaard et & $6.41 \%$ & $6.45 \%$ & $6.37 \%$ & 0.03 \\
al 2019 & & & \\
& & & \\
& & & \\
Warmelink & $23.22 \%$ & $24.03 \%$ & $22.45 \%$ & 0.36 \\
et al 2012 & & & & {$[-0.07,0.79]$} \\
& & & & 0.24 \\
Mac Giolla & $1.53 \%$ & $1.68 \%$ & $1.39 \%$ & {$[-0.16,0.64]$} \\
et al 2019 & & & & 0.70 \\
Verschuere & $13.42 \%$ & $14.48 \%$ & $12.24 \%$ & {$[0.21,1.18]$} \\
et al 2021 & & & & \\
\hline
\end{tabular}

Second, we reconverted LIWC proportion scores to count scores, allowing for a direct comparison with the manual count scores. LIWC count scores were calculated by [statement word count] * [proportion score of LIWC category] / 100. Table 4 shows that lie-truth effect sizes for LIWC count scores varied from $-0.09 \leq d \leq 0.50$ and was statistically significant in two of the four datasets. When both manual and LIWC scores were expressed as count scores, neither method seemed to systematically outperform the other, see Table 1 and 4.

Table 4. Perceptual and contextual details in truthful vs deceptive statements: LIWC count scores 


\begin{tabular}{|c|c|c|c|c|}
\hline & $\begin{array}{c}\text { Across } \\
\text { statements }\end{array}$ & $\begin{array}{c}\text { True } \\
\text { statemen }\end{array}$ & $\begin{array}{c}\text { Lie } \\
\text { statement }\end{array}$ & $\begin{array}{l}\text { Lie-truth Differences } \\
\text { Cohen's d }[95 \% \mathrm{CI}]\end{array}$ \\
\hline & & ts & $\mathrm{s}$ & \\
\hline Bogaard et & 26.53 & 29.07 & 24.00 & 0.25 \\
\hline al 2019 & $(20.25)$ & (22.86) & (16.98) & {$[0.06,0.43]$} \\
\hline Warmelink & 157.25 & 172.80 & 142.41 & 0.20 \\
\hline et al 2012 & $(148.65)$ & $(182.18)$ & (107.58) & {$[-0.23,0.63]$} \\
\hline Mac Giolla & 24.36 & 23.71 & 25.00 & -0.09 \\
\hline et al 2019 & $(14.85)$ & (15.00) & $(14.84)$ & {$[-0.49,0.31]$} \\
\hline Verschuere & 35.94 & 40.68 & 30.65 & 0.50 \\
\hline et al 2021 & $(20.61)$ & (23.00) & $(16.31)$ & {$[0.02,0.98]$} \\
\hline
\end{tabular}

The impression that, when using the same metric, neither method seemed to systematically outperform the other is confirmed by the results of a series of 2 (Veracity: truth vs lie) x 2 (Method: Manual vs LIWC) Bayesian ANOVAs that we ran for each dataset and each metric (count scores and proportions), separately (so 4 datasets $\mathrm{x} 2$ metrics $=8$ ANOVAs). Specifically, using JASP 0.13.1.0 and its default settings, and following the JASP guidelines (Wagenmakers et al., 2017), we looked at the extent to which the data supported the inclusion of the interaction term as compared to the simple main effects of Veracity and Method (i.e., the model with Veracity, Method, and their interaction versus the model with only Veracity and Method). The Bayes factor $\left(\mathrm{BF}_{10}\right)$ indicates how much more likely the data are under the 
hypothesis of only the two main effects versus the addition of their interaction. Table 5 shows there is never strong support for the addition of the interaction term, and for 7 out of 8 comparisons the data provide some evidence against the inclusion of the interaction term.

Table 5. The evidential value $\left(\mathrm{BF}_{10}\right)$ that manual vs LIWC coding differ in their ability to differentiate lies from truths (i.e., $\mathrm{BF}_{10}{ }^{\prime} \mathrm{s}<1$; indicating that the data are more likely under the null hypothesis of no interaction than under the interaction hypothesis).

\begin{tabular}{lcc}
\hline & $\begin{array}{c}\text { Count scores } \\
\left(\mathrm{BF}_{10}\right)\end{array}$ & Proportions \\
& 0.48 & $\left(\mathrm{BF}_{10}\right)$ \\
\hline Bogaard et al 2019 & 0.22 & 0.15 \\
Warmelink et al 2012 & 0.52 & 0.20 \\
Mac Giolla et al 2019 & & 2.97 \\
Verschuere et al 2021 & 0.35 & 0.25 \\
\hline
\end{tabular}

\section{Correlations between manual and LIWC coded perceptual and contextual details}

Table 6, first column, shows that the correlation between manual count scores and LIWC proportion scores is low, $0.09 \leq r \leq 0.30$. This points to the possibility that LIWC and manual coding do not measure the same construct. The low association may also be related to the fact that LIWC relies on proportion scores and manual coding on count scores. The second and third columns therefore show the correlations when using the same metric (count scores, and proportion scores, respectively). The high correlations between manual and LIWC count scores, $0.55 \leq r \leq 0.90$, may be largely a result of statement length. Relying on the same metric 
and controlling for statement length, the correlations between manual and LIWC proportion scores, $0.08 \leq r \leq 0.48$, may best reflect the (weak to modest) correspondence between manual and LIWC coding.

Table 6. Pearson Correlations between manual and LIWC coded perceptual and contextual details

\begin{tabular}{|c|c|c|c|}
\hline & $\begin{array}{l}\text { Manual count scores, } \\
\text { LIWC proportion scores } \\
\text { (with } 95 \% \mathrm{CI} \text { ) }\end{array}$ & $\begin{array}{l}\text { Manual count scores, } \\
\text { LIWC count scores } \\
\text { (with } 95 \% \mathrm{CI})\end{array}$ & $\begin{array}{c}\text { Manual proportion scores, } \\
\text { LIWC proportion scores } \\
\text { (with } 95 \% \mathrm{CI})\end{array}$ \\
\hline Bogaard et al & $0.30 * * *$ & $0.68 * * *$ & $0.31 * * *$ \\
\hline 2019 & $(0.17,0.42)$ & $(0.60,0.75)$ & $(0.18,0.43)$ \\
\hline Warmelink et & 0.19 & $0.90 * * *$ & 0.08 \\
\hline al 2012 & $(-0.02,0.39)$ & $(0.84,0.93)$ & $(-0.13,0.29)$ \\
\hline Mac Giolla et & 0.09 & $0.55 * * *$ & $0.48 * * *$ \\
\hline al 2019 & $(-0.11,0.29)$ & $(0.40,0.68)$ & $(0.31,0.62)$ \\
\hline Verschuere et & 0.14 & $0.90 * * *$ & $0.48 * * *$ \\
\hline al 2021 & $(-0.09,0.36)$ & $(0.85,0.94)$ & $(0.28,0.64)$ \\
\hline
\end{tabular}

Note: ${ }^{*}$ denotes $p<.05 ; * *$ denotes $p<.01, * * *$ denotes $p<.001$

Variation in manual coding

Finally, we observed great differences in manual coding between datasets. To illustrate, the proportion of the statement that is marked as a detail, varied from $1.53 \%$ to $23.22 \%$, see 
Table 3. To illustrate the difference in manual coding, we asked each lab to code one example statement, and we also LIWC coded it, see Appendix I.

\section{General Discussion}

Verbal lie detection typically relies on manual coding of statements, with perceptual and contextual details being amongst the most prevalently used cues to deceit (Vrij, 2019). There exists, however, no standardized way of coding, and coding can differ greatly between human coders (Nahari et al., 2019). While an automated approach may resolve concerns regarding subjectivity in coding (i.e. improve the reliability of the approach), the only study to date to directly compare automated to manual coding of RM criteria found manual coding to be superior (Vrij et al., 2007). Here, we expand the man vs machine comparison to four other datasets.

The validity of manually counted perceptual and contextual details in discriminating lie from truth varied substantially across the four datasets. Significant lie-truth differences were found for two datasets, and substantial differences only for one of the four datasets. This fits with meta-analytic conclusions that even the most discriminative cues on average show only small to moderate effects (DePaulo et al., 2003; Luke, 2019). Moreover, these meta-analytic investigations found considerable variation between studies in effect sizes. A factor that may explain variation in the current set of studies is whether statements were about past or future experiences. It is noteworthy that we found significant effects for the statements about past events (the Bogaard et al., 2019 and Verschuere et al., 2021 datasets) but not those about intentions (the Mac Giolla et al, 2019 and Warmelink et al., 2012 datasets). Reality Monitoring theory was initially developed for past actions and may not readily apply to future actions (for a discussion see Mac Giolla et al, 2019), which may explain the higher validity in the statements about past events than those about future events. 
Automated coding of perceptual and contextual details (here: LIWC proportion scores) showed substantial differences in validity, with significant lie-truth effects for two out of four datasets (i.e., those about intentions; MacGiolla et al 2019 and Warmelink et al., 2012). It is not immediately clear why LIWC would perform better for statements about intentions, and other differences between the studies could drive the apparent differences. Moreover, when reconverting LIWC proportion scores to LIWC count scores, the results were more in line with the manual count scores: Significant lie-truth effects for LIWC count scores were found for the past event but not the future event statements. Interestingly, even small to moderate average effects of automated coding could be of interest for the study of deception. Small to moderate effects of automated coding may even be of use for lie detection purposes when combined with other cues (e.g., Fitzpatrick, Bachenko, \& Fornaciari, 2015; Kleinberg, van der Toolen, Vrij, Arntz, \& Verschuere, 2018; Ott, Choi, Cardie, \& Hancock, 2011). Furthermore, computer coding can be fully automated. Not just the coding itself, but also the transcription of the (oral) statements, and even the conclusions (e.g., truth probability; see Kleinberg and Verschuere, 2021). For some types of automated decision making - for instance, when based on neural network approaches - it is no longer clear how the computer comes to its lie-truth classification. Such an automated approach raises important questions with regard to transparency, interpretability and ultimately accountability (Barredo Arrieta et al., 2020), and may be deemed undesirable when having real-life consequences (e.g., in the courtroom).

We also found that manual coding practices differed greatly between the four laboratories. Appendix I clearly illustrates how large those differences are (see also Masip et al., 2005; Kleinberg, Arntz, \& Verschuere, 2019). Notably, some ways of coding score a much greater proportion of the statement as a detail than others, and the incremental validity compared to simple word count is challenged (see also Elntib et al., 2015; Elntib \& Wagstaff, 2017; Masip et al., 2005). This calls for default reporting of effects of statement length in papers 
relying on manual coding (Lange, 2019). Moreover, while many researchers agree that some measure of "detail" is a dependent variable of interest (see e.g., Luke, 2019), there is no generally agreed upon definition of this concept and hence no standardized operationalization. This allows different labs to use different definitions, compounding the difficulty of measuring them accurately. The development of a validated and widely accepted definition of details should be a priority for researchers using details for truth-lie discrimination.

This study is not without limitations. First, the search and selection of the datasets was not systematic. Future research would benefit from using an open and systematic search for existing datasets and/or dedicated new data collection. Methodological examinations like the current one rely on data availability. Making the (anonymized or pseudonymized) statements available upon publication would make the validation of scoring methods more efficient. Second, the datasets differ in several aspects including language, event type, interview type, and coding scheme, hampering our ability to isolate the factors that may explain differences between automated and manual verbal lie detection accuracy. Comparisons of manual with automated approaches on the same set of statements would benefit many deception studies. Third, the number of words recognized by LIWC differed greatly between languages. While $86 \%$ and $94 \%$ of the words were recognized by the LIWC in the Dutch and English versions, respectively, only 55\% of Swedish words were recognized. Thus, the Swedish LIWC dictionary needs expansion to provide a more comprehensive automated coding of Swedish texts. In addition, this study focussed on a direct comparison between manual coding and LIWC coding, since the dictionary approach allowed for a word-by-word comparison. Yet there are other tools available in the field of automated text analysis, based on different approaches (Grimmer \& Stewart, 2013).

In sum, because the comparison of automated with manual coding has relied mostly on a between-study comparison, and the two methods rely on different metrics (manual count 
scores vs LIWC proportion scores), it may have been premature to dismiss automated coding (Vrij, 2008; Vrij et al., 2007). When compared on the same metric, neither method consistently outperformed the other. And when relying on count scores, both LIWC and manual coding suggest that truthful statements about past events contain more perceptual and contextual details than deceptive statements. Making datasets of manually coded statements publicly available will allow for a systematic, large-scale, and direct comparison of manual and automated approaches and inform whether and when perceptual and contextual details allow for lie-truth discrimination. 


\section{References}

Bogaard, G., Colwell, K., \& Crans, S. (2019). Using the Reality Interview improves the accuracy of the Criteria-Based Content Analysis and Reality Monitoring. Applied Cognitive Psychology. https://doi.org/10.1002/acp.3537

Bond, G. D., \& Lee, A. Y. (2005). Language of lies in prison: Linguistic classification of prisoners' truthful and deceptive natural language. Applied Cognitive Psychology, 19(3), 313-329. https://doi.org/10.1002/acp.1087

DePaulo, B. M., Lindsay, J. J., Malone, B. E., Muhlenbruck, L., Charlton, K., \& Cooper, H. (2003). Cues to deception. Psychological bulletin, 129(1), 74.

Elntib, S., \& Wagstaff, G. (2017). Are reality monitoring differences between truthful and deceptive autobiographical accounts affected by standardisation for word-count and the presence of others? Psychology, Crime and Law, 23(7), 699-716. https://doi.org/10.1080/1068316X.2017.1298762

Elntib, S., Wagstaff, G. F., \& Wheatcroft, J. M. (2015). The Role of Account Length in Detecting Deception in Written and Orally Produced Autobiographical Accounts using Reality Monitoring. Journal of Investigative Psychology and Offender Profiling, 12(2), 185-198. https://doi.org/10.1002/jip.1420

Fitzpatrick, E., Bachenko, J., \& Fornaciari, T. (2015). Automatic Detection of Verbal Deception. Synthesis Lectures on Human Language Technologies, 8(3), 1-121. https://doi.org/10.2200/S00656ED1V01Y201507HLT029

Gibbons, R. D., Hedeker, D. R., \& Davis, J. M. (1993). Estimation of effect size from a series of experiments involving paired comparisons. Journal of Educational Statistics, 18, 271-279. 
Grimmer, J., \& Stewart, B. M. (2013). Text as data: The promise and pitfalls of automatic content analysis methods for political texts. Political analysis, 21(3), 267-297.

Hauch, V., Blandón-Gitlin, I., Masip, J., \& Sporer, S. L. (2015). Are computers effective lie detectors? A meta-analysis of linguistic cues to deception. Personality and Social Psychology Review, 19(4), 307-342.

Johnson, M. K., \& Raye, C. L. (1981). Reality monitoring. Psychological Review, 88(1), 67-85. https://doi.org/10.1037/0033-295X.88.1.67

Kleinberg, B., Arntz, A., \& Verschuere, B. (2019). Being accurate about verbal credibility assessment, 1-13. https://doi.org/10.31234/OSF.IO/H6PXT

Kleinberg, B., van der Toolen, Y., Vrij, A., Arntz, A., \& Verschuere, B. (2018). Automated verbal credibility assessment of intentions: The model statement technique and predictive modeling. Applied Cognitive Psychology, 32(3), 354-366. https://doi.org/10.1002/acp.3407

Lange, F. (2019). Are Difficult-To-Study Populations too Difficult to Study in a Reliable Way? Lessons Learned From Meta-Analyses in Clinical Neuropsychology. European Psychologist. https://doi.org/10.1027/1016$9040 / \mathrm{a} 000384$.

Luke, T. J. (2019). Lessons From Pinocchio: Cues to Deception May Be Highly Exaggerated. Perspectives on Psychological Science, 14(4), 646-671. https://doi.org/10.1177/1745691619838258

Mac Giolla, E., Ask, K., Granhag, P. A., \& Karlsson, A. (2019). Can Reality Monitoring Criteria Distinguish Between True and False Intentions? Journal of Applied Research in Memory and Cognition, 8(1), 92-97. https://doi.org/10.1016/j.jarmac.2018.08.002 
Masip, J., Sporer, S. L., Garrido, E., \& Herrero, C. (2005). The detection of deception with the reality monitoring approach: a review of the empirical evidence. Psychology, Crime \& Law, 11(1), 99-122. https://doi.org/10.1080/10683160410001726356

Nahari, G. (2018). The Applicability of the Verifiability Approach to the Real World. In Detecting Concealed Information and Deception: Recent Developments (pp. 329 349). Elsevier. https://doi.org/10.1016/B978-0-12-812729-2.00014-8

Nahari, G., Ashkenazi, T., Fisher, R. P., Granhag, P. A., Hershkowitz, I., Masip, J., ... Vrij, A. (2019). 'Language of lies': Urgent issues and prospects in verbal lie detection research. Legal and Criminological Psychology, 24(1), 1-23. https://doi.org/10.1111/lcrp.12148

Ott, M., Choi, Y., Cardie, C., \& Hancock, J. T. (2011, June). Finding deceptive opinion spam by any stretch of the imagination. In Proceedings of the 49th annual meeting of the association for computational linguistics: Human language technologiesvolume 1 (pp. 309-319). Association for Computational Linguistics.

Pennebaker, J. W., Boyd, R. L., Jordan, K., \& Blackburn, K. (2015). The development and psychometric properties of LIWC2015. Austin, TX: University of Texas at Austin.

Torchiano, M. (2019). effsize: Efficient Effect Size Computation. doi: 10.5281/zenodo.1480624, R package version 0.7.6, https:/CRAN.Rproject.org/package=effsize.

Van Wissen, L., \& Boot, P. (2017, September). An electronic translation of the LIWC Dictionary into Dutch. In Electronic lexicography in the 21st century: Proceedings of eLex 2017 conference (pp. 703-715). Lexical Computing. 
Vrij, A. (2008). Detecting lies and deceit: Pitfalls and opportunities. Hoboken, US: John Wiley \& Sons, Ltd.

Vrij, A. (2019). Deception and truth detection when analyzing nonverbal and verbal cues. Applied Cognitive Psychology, 33(2), 160-167. https://doi.org/10.1002/acp.3457

Vrij, A., Mann, S., Kristen, S., \& Fisher, R. P. (2007). Cues to deception and ability to detect lies as a function of police interview styles. Law and Human Behavior, 31(5), 499-518. https://doi.org/10.1007/s10979-006-9066-4

Warmelink, L., Vrij, A., Mann, S., Jundi, S., \& Granhag, P. A. (2012). The effect of question expectedness and experience on lying about intentions. Acta Psychologica, 141(2), 178-183. https://doi.org/10.1016/j.actpsy.2012.07.011 
Appendix I. Example statement from the Warmelink et al. 2012 dataset, coded with LIWC and by humans (Mac Giolla et al 2019: coded by author EM; Warmelink et al 2019; coded by author LW, Bogaard et al 2019; Verschuere et al 2021: coded by author MS) for perceptual and contextual details.

\begin{tabular}{|c|c|c|c|c|c|}
\hline & LIWC coding & $\begin{array}{l}\text { Manual coding } \\
\text { Warmelink et al } \\
2019\end{array}$ & $\begin{array}{l}\text { Manual coding } \\
\text { Mac Giolla et al } \\
2019\end{array}$ & $\begin{array}{l}\text { Manual coding } \\
\text { Bogaard et al } \\
2019\end{array}$ & $\begin{array}{l}\text { Manual coding } \\
\text { Verschuere et al } \\
2021\end{array}$ \\
\hline $\begin{array}{l}\text { Question was } \\
\text { 'How are } \\
\text { you going to } \\
\text { travel to your } \\
\text { destination?' }\end{array}$ & $\begin{array}{l}\text { Err Euro Star, and } \\
\text { then... well Euro } \\
\text { Star to erm Paris, } \\
\text { then Paris to } \\
\text { Cologne... stay } \\
\text { overnight in } \\
\text { Cologne and then } \\
\text { we go it says high } \\
\text { speed train to } \\
\text { Austria. Now it } \\
\text { doesn't look that } \\
\text { far on the map } \\
\text { between Cologne } \\
\text { and Austria, but I } \\
\text { think it takes about } \\
7 \text { hours (laughs) } \\
\text { so I don't know if } \\
\text { it goes through the } \\
\text { mountains, and if }\end{array}$ & $\begin{array}{l}\text { Err Euro Star, } \\
\text { and then... well } \\
\text { Euro Star to erm } \\
\text { Paris, then Paris } \\
\text { to Cologne... stay } \\
\text { overnight in } \\
\text { Cologne and } \\
\text { then we go it } \\
\text { says high speed } \\
\text { train to Austria. } \\
\text { Now it doesn't } \\
\text { look that far on } \\
\text { the map between } \\
\text { Cologne and } \\
\text { Austria, but I } \\
\text { think it takes } \\
\text { about } 7 \text { hours } \\
\text { (laughs) so I don't } \\
\text { know if it goes }\end{array}$ & $\begin{array}{l}\text { Err Euro Star, and } \\
\text { then... [well Euro } \\
\text { Star to erm } \\
\text { Paris], [then Paris } \\
\text { to Cologne]... stay } \\
\text { [overnight in } \\
\text { Cologne] and } \\
\text { [then we go it } \\
\text { says] [high speed } \\
\text { train] to Austria. } \\
\text { Now it doesn't } \\
\text { look that far on the } \\
\text { map between } \\
\text { Cologne and } \\
\text { Austria, but I think } \\
\text { it takes about } 7 \\
\text { hours (laughs) so I } \\
\text { don't know if it } \\
\text { goes through the }\end{array}$ & $\begin{array}{l}\text { Err Euro Star, } \\
\text { and then... well } \\
\text { Euro Star to erm } \\
\text { Paris, then Paris } \\
\text { to Cologne... stay } \\
\text { overnight in } \\
\text { Cologne and then } \\
\text { we go it says high } \\
\text { speed train to } \\
\text { Austria. Now it } \\
\text { doesn't [look that } \\
\text { far on the map } \\
\text { between Cologne } \\
\text { and Austria], but } \\
\text { I think it takes } \\
\text { about } 7 \text { hours } \\
\text { (laughs) so I don't } \\
\text { know if it goes } \\
\text { through the }\end{array}$ & $\begin{array}{l}\text { Err Euro Star, } \\
\text { and then... well } \\
\text { Euro Star to erm } \\
\text { Paris, then Paris } \\
\text { to Cologne... stay } \\
\text { overnight in } \\
\text { Cologne and then } \\
\text { we go it says high } \\
\text { speed train to } \\
\text { Austria. Now it } \\
\text { doesn't look that } \\
\text { far on the map } \\
\text { between Cologne } \\
\text { and Austria, but I } \\
\text { think it takes } \\
\text { about } 7 \text { hours } \\
\text { (laughs) so I don't } \\
\text { know if it goes } \\
\text { through the }\end{array}$ \\
\hline
\end{tabular}




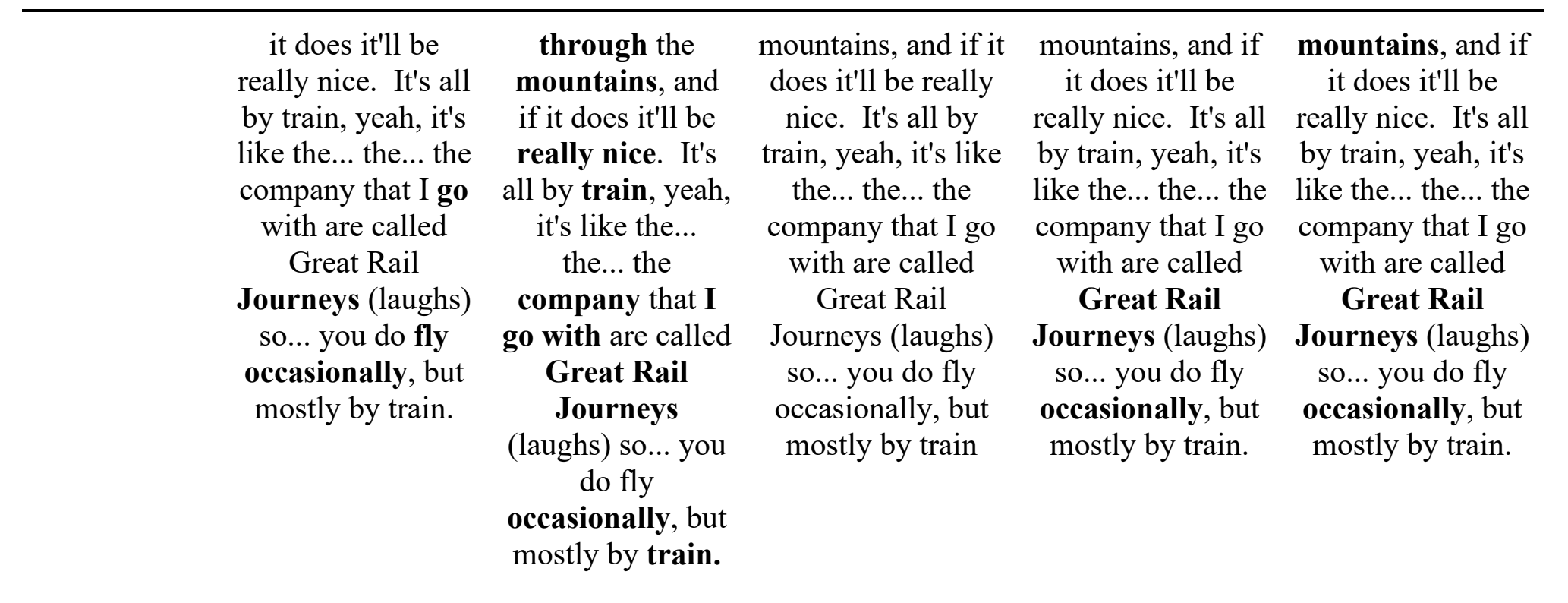

\footnotetext{
Note: Perceptual and contextual details are marked in bold. LIWC uses a dictionary approach hence does not recognize all words, which may

explain why city of Cologne, but not Paris is marked as a contextual detail.
} 
